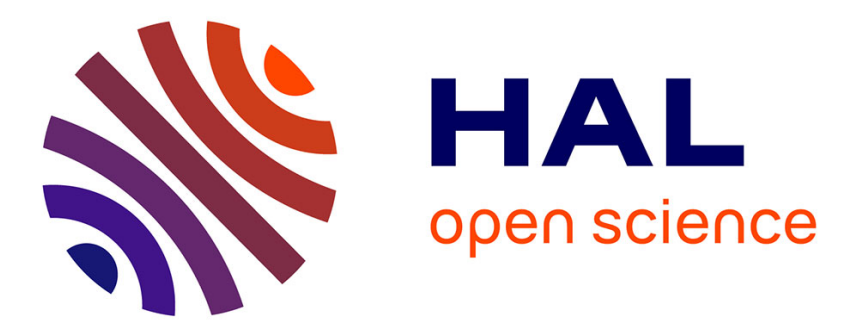

\title{
Insuffisance rénale aiguë obstructive : une lecture physiopathologique
}

Jean-Philippe Haymann, Christophe Vinsonneau, Alexis Girshovich, Michel Daudon

\section{- To cite this version:}

Jean-Philippe Haymann, Christophe Vinsonneau, Alexis Girshovich, Michel Daudon. Insuffisance rénale aiguë obstructive : une lecture physiopathologique. Néphrologie \& Thérapeutique, 2017, 13 (Suppl. 1), pp.S1-S5. 10.1016/j.nephro.2017.01.008 . hal-01534519

\section{HAL Id: hal-01534519 https: / hal.sorbonne-universite.fr/hal-01534519}

Submitted on 7 Jun 2017

HAL is a multi-disciplinary open access archive for the deposit and dissemination of scientific research documents, whether they are published or not. The documents may come from teaching and research institutions in France or abroad, or from public or private research centers.
L'archive ouverte pluridisciplinaire HAL, est destinée au dépôt et à la diffusion de documents scientifiques de niveau recherche, publiés ou non, émanant des établissements d'enseignement et de recherche français ou étrangers, des laboratoires publics ou privés. 
Insuffisance rénale aiguë obstructive : Une lecture physiopathologique

Acute obstructive nephropathy: A pathophysiological view

Jean-Philippe Haymann ${ }^{a, b, c,}$, Christophe Vinsonneau ${ }^{b}$, Alexis Girshovich ${ }^{b}$, Michel Daudon ${ }^{a, b}$

${ }^{a}$ Service d'explorations fonctionnelles multidisciplinaires, hôpital Tenon, 4, rue de la Chine, 75020 Paris, France

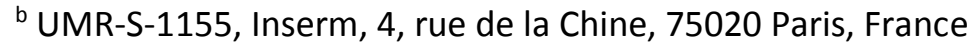

c UMR-S-1155, université Pierre-et-Marie-Curie, 4, rue de la Chine, 75020 Paris, France

Jean-philippe.haymann@aphp.fr 


\section{Résumé}

L'insuffisance rénale aigue obstructive apparaît une entité hétérogène, la physiopathologie des obstructions intra tubulaires étant différente de celle des obstructions aiguës des voies excrétrices. En effet dans un cas les tubules sont dilatés et le régime de pression dans les uretères, bassinets et calices est normal, dans l'autre cas, les contraintes exercées sur les parois sont importantes et entraînent une déformation des cavités rénales conduisant rapidement à une compression extrinsèque du système tubulaire avec des tubules généralement pas ou peu dilatés, les pressions intra tubulaires étant néanmoins élevées. L'insuffisance rénale aigue obstructive est cependant dans tous les cas une insuffisance rénale aigue organique. La présence de cristaux, de cellules desquamées, de débris cellulaires et/ou de gels protéiques intratubulaires entraine une prolifération tubulaire de voisinage, un recrutement inflammatoire et, dans les cas favorables, une résolution. Cependant la présence de séquelles à type de fibrose est une éventualité qui ne doit pas faire sousestimer la fréquence d'un défaut de reperméabilisation des lumières tubulaires avec constitution de nombreux néphrons non fonctionnels du fait de glomérules borgnes (sans tubule). Les phénomènes d'autorégulation de l'hémodynamique rénale ne sont donc pas nécessairement destinés uniquement à préserver un débit sanguin rénal constant mais aussi à maintenir un flux tubulaire afin d'éviter l'interruption définitive du flux par une prolifération anarchique des cellules tubulaires. La survenue d'une obstruction tubulaire focale et le diagnostic de néphropathies cristallines semble sous-estimée au cours de la maladie rénale chronique et suggère l'intérêt thérapeutique potentiel de certains solubilisateurs ou inhibiteurs macromoléculaires en aigue et en chronique.

Mots clés: Insuffisance rénale aigue obstructive, physiopathologie, néphropathie cristalline, compression extrinsèque, inhibiteurs macromoléculaires de solubilisation, glomérule atubulaire. 


\section{Summary}

Obstructive acute renal failure is a heterogeneous entity as the pathophysiology of intratubular obstruction is quite different from upper tract obstruction. In the former case, tubules are dilated due to a high hydrostatic pressure whereas pressures are normal in urinary upper tract. In the latter case, a high pressure above the ureteral obstacle is responsible for dilated renal cavities leading to extrinsic compression with no or only few dilated tubules though high hydrostatic pressure are recorded within tubules. Obstruction within tubules may be related to crystal formation, exfoliated cells, cellular debris and/or protein gels altogether with cell proliferation, and recruitment of inflammatory cells. Though fibrosis may develop, occurrence of atubular glomeruli in several nephrons due to an initial loss of tubular patency highlights the critical importance of maintaining a fluid flow within tubules in order to avoid uncontrolled tubular cell proliferation. The onset of tubular obstruction in few tubules especially in crystal nephropathy is underestimated especially in chronic kidney disease patients, thus suggesting that some macromolecular solubilizing factors may be potential relevant therapy to prevent (and/or reverse) chronic kidney disease progression or decrease acute renal failure sequelae.

Key words: Obstructive acute renal failure, pathophysiology, crystal nephropathy, extrinsic compression, macromolecular inhibitors, atubular glomeruli 
Abbreviations

Insuffisance rénale aigue (IRA)

Tube contourné proximal (TCP)

Débit sanguin rénal (DSR)

Débit de filtration glomérulaire (DFG) 


\section{A) Introduction}

Les insuffisances rénales aigues (IRA) sont classiquement divisées en IRA fonctionnelles, IRA organiques et IRA obstructives. Cette distinction en grandes catégories est fondée sur une approche empirique permettant une conduite à tenir schématique utile pour les étudiants : hydratation par un soluté salé, réalisation d'une ponction biopsie rénale ou drainage urinaire respectivement. Les IRA obstructives sont en effet habituellement assimilées à un obstacle des voies urinaires responsable d'une dilatation des cavités excrétrices visibles à l'échographie, à la tomodensitométrie ou à l'IRM. La liste des IRA obstructives comprend néanmoins des causes intratubulaires, situation donc assimilable à des IRA organiques.

Les obstacles tubulaires peuvent être de nature très différente: intrinsèque comme dans les précipitations cristallines ou la formation de cylindres et gels proteiques au décours de lésions tubulaires (ischémiques, toxiques...) ; mais aussi extrinsèque, par exemple au cours des multi ou polykystoses, les kystes comprimant les tubules de voisinage. Le mécanisme d'obstruction est donc un phénomène présent dans de très nombreuses pathologies, et probablement sous-estimé notamment en ce qui concerne les néphropathies cristallines (1). Par ailleurs, de nombreuses IRA médicamenteuses sont souvent à tort classées comme IRA toxiques ou immuno allergiques alors qu'il s'agit en fait d'une néphropathie cristalline.

Du fait de différences notables dans les mécanismes mis en jeu, une approche physiopathologique des IRA obstructives consiste donc à distinguer les obstacles par précipitations intra tubulaires des obstacles des voies excrétrices.

\section{B) IRA par obstacle intratubulaire}

La formation de bouchons protéiques, cristallins et/ou médicamenteux intra tubulaires est habituellement évitée du fait d'un flux tubulaire conséquent avec une faibles présence de protéines 
ou de métabolites exogènes (médicamenteux) et d'une sursaturation en solutés inférieure aux concentrations nécessaires à la formation rapide de germes cristallins oxalo-calciques et phosphocalciques, mais aussi uratiques, cystiniques.... Habituellement, si quelques cristallites se forment dans le néphron du fait d'un allongement du temps de transit ou d'une sursaturation élevée dans un segment du néphron, la mise en jeu de mécanismes efficaces permet d'éliminer ces cristallites dans les cavités excrétrices sans entraîner d'obstruction tubulaire. A l'inverse, on comprend qu'une précipitation massive puisse survenir au cours d'une IRA du fait d'un effondrement de la filtration glomérulaire et donc du flux tubulaire notamment lorsque une souffrance tubulaire existe. L'obstruction tubulaire survient cependant préférentiellement dans certains segments du néphron.

\section{I) Localisation des dépôts cristallins, protéiques \& médicamenteux}

\section{Le canal collecteur}

En théorie, le canal collecteur apparaît comme le segment du néphron le plus à risque d'obstruction, le débit urinaire étant le plus faible lorsque le patient est en antidiurèse. De plus dans la partie médullaire du canal collecteur, en situation d'antidiurèse, l'osmolalité intra-tubulaire est trois fois plus élevée que dans le plasma (2). Ce segment est donc théoriquement plus à risque. Ce risque est d'autant plus élevé que le $\mathrm{pH}$ intra tubulaire en situation physiologique varie entre 4.5 et 7.0 au cours du nycthémère et que toute condition minimisant ses variations augmente le risque de cristallisation des espèces fortement dépendantes du $\mathrm{pH}$ : I'acide urique lorsque le $\mathrm{pH}$ est stable et $<5.5$ ou diverses variétés de phosphates de calcium lorsque le pH est alcalin>6.8- 7.0.

\section{Le tube contourné proximal (TCP)}

Le TCP est un segment théoriquement peu susceptible d'obstruction tubulaire si l'on considère l'importance du débit tubulaire, le court temps de transit et un pH aux alentours de 6.9 à la fin de ce segment après la réabsorption d'une majeure partie des bicarbonates filtrés. Aussi, la survenue 
d'une cristallisation s'observe principalement dans ce segment S3 où le calibre de la lumière tubulaire est le plus étroit, lors d'une augmentation importante de la charge filtrée de substances cristallisables comme par exemple lors d'une intoxication à l'éthylène glycol (qui est rapidement métabolisé en oxalates). Un tel processus reproduit dans un modèle expérimental (3) dépend en effet de la sursaturation en oxalates dans la lumière tubulaire. Une sursaturation importante permet une germination nombreuse, une croissance et une agrégation cristalline rapide de cristallites, composés d'oxalates de calcium monohydratés dont la forme est lancéolées (4). La géométrie des agrégats, notamment la taille et la forme sont ici probablement des éléments déterminants facilitant l'obstruction tubulaire mais aussi probablement également l'accrochage à la bordure en brosse des cellules du TCP.

\section{Autres segments tubulaires}

Une obstruction peut en effet être retrouvée dans tous les segments du tubule comme le montre le modèle expérimental d'IRA par administration de myoglobine (5). En effet dans ce modèle, les cylindres de myoglobine sont présents dans la branche fine de l'anse Henle et les canaux collecteurs médullaires et papillaires, mais une nécrose tubulaire avec desquamation et obstruction tubulaire est retrouvée également dans les TCP, les branches de l'anse de Henle et les canaux collecteurs. Enfin, en dehors de ces phénomènes de compression intrinsèque par précipitation intra tubulaire cristalline et/ou protéique, des compressions extrinsèques locales sont susceptible de survenir dans la médullaire rénale probablement du fait de l’augmentation de la pression interstitielle.

\section{II) Rétention des cristaux, phénotype tubulaire et rôle des gels protéiques}

La fixation des cristaux à la lumière apicale des cellules tubulaires est facilitée par une modification du phénotype des cellules tubulaires qui deviennent « collantes » en réponse à des lésions induites par des cristaux ou pré-existantes et/ou au cours d'une régénération cellulaire (6). Cet accrochage 
est en effet rendu possible par la surexpression ou l'expression de novo de différents inhibiteurs macromoléculaires qui habituellement inhibent l'agrégation des cristallites, mais qui lors d'une sursaturation massive au contraire forment une " colle » et facilitent la formation d'agrégats de grande taille. Le travail de Vervaet et coll. (6) montre en effet l'augmentation de l'annexine 2 ou de la sécrétion de l'ostéopontine et de l'acide hyaluronique ainsi que la surexpression tubulaire de leur récepteur membranaire apical le CD44 chez l'homme ou l'animal au cours des néphropathies cristallines. Ces phénomènes à l'évidence favorisent l'adhésion des cristaux aux tubules i.e. donc leur immobilisation avec la conséquence d'une diminution de calibre de la lumière et un ralentissement du flux jusqu'à obstruction totale.

Un certain nombre de macromolécules sont sécrétées dans la lumière tubulaire en physiologie mais surtout en grande quantité au cours d'un processus lésionnel (7). C'est le cas notamment de I'uromoduline ou de l'ostéopontine qui contribuent en fonction de l'environnement physico chimique à la formation d'un gel protéique avec constitution des cylindres qui obstruent la lumière tubulaire $(7,8)$ et qui sont retrouvés dans les urines ultérieurement lors de la "guérison ». Ce processus d'agrégation et/ou de gélification peut également se produire en présence de certaines protéines anormales filtrées par les glomérules, situation rencontrée au cours de certains myélomes à chaîne légère (9).

\section{III) Influence d'une obstruction tubulaire sur les pressions intraglomérulaires et intratubulaires}

Des études sur néphrons isolés menées chez le rat dans les années 1970 ont permis de montrer que l'obstruction tubulaire était un événement qui survenait très précocement après une interruption du flux sanguin rénal (10). Par ailleurs, dans les travaux de Arendhorst et coll. (10), 24 heures après la constitution d'un obstacle tubulaire (réalisé par injection dans un tube distal d'une goutte d'huile), la pression capillaire glomérulaire diminue de 38 à $26 \mathrm{mmHg}$ alors que dans le même temps la pression intratubulaire en amont de l'obstacle augmente de 11.9 à $15.7 \mathrm{mmHg}$. La diminution importante de la pression de filtration effective, 24 heures après une obstruction, est donc expliquée non 
seulement par une augmentation de la pression hydrostatique intratubulaire, mais également par une vasoconstriction de l'artériole afférente (10).

Dans un modèle expérimental d'IRA obstructive par précipitation de lysine intratubulaire chez le rat (11), la pression intratubulaire augmente dès les premières 90 minutes et s'accompagne d'une dilatation des tubules significative (l'augmentation secondaire de diamètre permettant une moindre augmentation de la pression), une diminution précoce du DFG de l'ordre de $50 \%$ mais sans modification du débit sanguin rénal (DSR) contrairement au modèle précédent. Une adaptation hémodynamique comportant une diminution du DSR avec augmentation des résistances intrarénales survient seulement à partir de la 3ème heure (11). Cette vasoconstriction retardée du système artériel rénal permet de diminuer, ainsi la pression de filtration et donc, dans une certaine mesure, le niveau de pression intratubulaire.

\section{IV) Influence d'une obstruction tubulaire sur les pressions dans la médullaire et la papille}

Contrairement au cortex, dans la papille et la médullaire interne, le flux sanguin rénal n'est pas ou peu soumis à une auto régulation (12). Cette région des reins est par ailleurs limitée par les espaces urinaires et ne peut donc que difficilement se déformer: une dilatation tubulaire des canaux collecteurs exercera pour cette raison une compression des structures de voisinage i.e. 1) des segments de l'anse de Henle avec rétrécissement de la lumière plus ou moins compensée par l'augmentation simultanée des pressions intraluminales dans les segments en amont (pour les néphrons soumis à une obstruction tubulaire ...) ;2) les vasa recta qui subiront une occlusion plus ou moins complète, empêchant ainsi la réabsorption tubulaire et donc contribuant à un œdème interstitiel d'où majoration de la pression interstitielle qui atteindra des valeurs positives de quelques mmHg (circonstance quasi unique dans l'organisme), facteur considéré comme essentiel dans le mécanisme de la pression de natriurèse (12) et expliquant principalement la néphropathie par perte de sel que l'on peut rencontrer au décours des levées d'obstacle. 


\section{C) IRA par obstacle dans les cavités excrétrices}

La mobilisation d'un calcul ou de tout autre obstacle dans l'uretère et son blocage dans le tractus urinaire avec interruption du flux urinaire, entraîne en quelques minutes une augmentation de pression en amont dans les cavités rénales qui se transmet également dans les tubes collecteurs jusque dans les chambres urinaires glomérulaires où les pressions peuvent atteindre $50 \mathrm{mmHg}$, alors que les valeurs habituelles sont de l'ordre de $4 \mathrm{mmHg}$ (13). Cette augmentation brutale des pressions hydrostatiques dans la chambre glomérulaire s'oppose à la pression de filtration et explique l'effondrement du débit de filtration glomérulaire (DFG). Au décours de cet obstacle, on observe la survenue d'une cascade d'événements :

1) Une réponse urétérale très rapide : l'augmentation de la pression en amont de l'obstacle entraine une augmentation de la tension pariétale de l'uretère expliquant une diminution du péristaltisme et la béance permanente de la lumière urétérale dans un délai d'environ 20 minutes (13). Après 1 heure, la pression intra luminale augmente de $7 \mathrm{mmHg}$ à environ $40 \mathrm{mmHg}$. A ce stade, le diamètre des uretères est augmenté mais il n'existe pas encore de " dilatation » : la géométrie « normale » de la lumière des uretères est conservée alors que les tensions pariétales sont élevées en permanence et non pas épisodiquement (comme observé lors des mouvements péristaltiques).

2) L’augmentation de la tension pariétale urétérale est transmise aux parois des cavités excrétrices intra rénales et au parenchyme rénal.

3) Puis une adaptation des uretères et des cavités rénales (bassinets et calices) à ce régime de pression inhabituel avec la survenue d'une dilatation permet ainsi de diminuer sensiblement la pression dans les cavités excrétrices. A ce stade, il existe donc une déformation du tissu rénal par compression extrinsèque : refoulement centrifuge du fait de la pression dans les cavités excrétrices qui est cependant limité par la capsule rénale. 
4) La réponse rénale est également précoce et comporte deux phases probablement en rapport avec la mécanique urétérale. On observe en effet au cours de la première heure une augmentation d'environ 30\% du débit sanguin rénal (DSR), probable mécanisme d'autorégulation du DFG mis en jeu afin de maintenir le DFG constant mais ici en pure perte du fait de l'obstacle complet urétéral. En fait, l'augmentation du DSR apparait ici comme un mécanisme délétère contribuant à majorer la pression dans les systèmes tubulaires et urinaires. Puis le DSR diminue progressivement d'environ $40 \%$ au cours des 24 heures qui suivent et correspond à l'augmentation des résistances intra rénales sélectivement dans le cortex (14), d'où une diminution attendue de la pression de filtration. Cette deuxième phase survient au moment de la dilatation des cavités excrétrices et contribue potentiellement à une normalisation relative des valeurs des pressions dans le système urinaire. En effet, la diminution des pressions de filtrations glomérulaires observée ne permet pas de production d'urine même si les pressions intra tubulaires (qui s'opposent à la filtration) diminuent sensiblement.

5) Un obstacle au drainage veineux survient probablement également rapidement au décours de l'obstacle urétéral en raison de la situation anatomique des vaisseaux lobaires qui sont localisés à proximité des espaces urinaires au niveau des calices notamment (figure 1D) (15). La compression extrinsèque des veines lobaires contribue potentiellement à l'apparition d'un œdème interstitiel, favorisé par ailleurs par l'augmentation de pression intra tubulaire, la diminution du DSR et l'augmentation des résistances intra rénales. Cet œdème interstitiel occupe potentiellement un volume supplémentaire qui contribue à aggraver la compression extrinsèque mentionnée plus haut.

Ces phénomènes mécaniques expliquent l'amincissement important du cortex rénal (figure $1 \mathrm{~A}$ et $\mathrm{B}$ ) observé dés $\mathrm{J} 7$ avec parfois disparition de la papille. Ce mécanisme de compression extrinsèque est conforté par une observation remarquable: la plupart des tubules ne sont pas dilatés. Les phénomènes biologiques engendrés par cette obstruction sont multiples (prolifération des cellules rénales résidentes, recrutement de cellules inflammatoires... et de nombreuses réactions chimiques 
induites par l'inflammation et les nécroses cellulaires) qui contribuent à la perte d'élasticité du parenchyme rénal et à sa destruction en l'absence de levée de l’obstacle (Figure 1B).

Curieusement, il a été noté expérimentalement une différence entre les IRA obstructives (obstructions urétérales bilatérales (BUO)) et les obstructions urétérales unilatérale (UUO). En effet, le nombre de néphrons fonctionnels est plus élevé dans l'UUO, après levée de l'obstacle urétéral à la 24ème heure. Une explication possible serait en rapport avec la constatation d'une abolition du reflexe tubulo-glomérulaire dans les IRA obstructives (BUO), responsable de pressions capillaires et intra tubulaires plus élevées alors que ce réflexe protecteur est au contraire stimulé dans les obstacles unilatéraux $(16,17)$.

\section{D) Mécanisme de résolution et de reperméabilisation tubulaire}

La diminution du DSR et de la pression de filtration observée dans les IRA par obstacle intratubulaire ou par obstacle dans les cavités excrétrices apparaît comme un phénomène retardé contribuant à diminuer le niveau de pression intra tubulaire, le flux étant interrompu du fait des obstacles en aval. Un régime de pression très élevé est en effet néfaste pour les cellules et peu compatible avec une régénération tubulaire (figure $1 \mathrm{C}$ ), les conséquences attendues étant davantage une desquamation cellulaire (18). Une sécrétion importante de macromolécules telles l'ostéopontine ou l'uromoduline (mais aussi la fibronectine, la vitronectine...) dans la lumière tubulaire constitue également un événement significatif. En effet ces molécules ont des propriétés chimiotactiques et pourraient permettre le homing de macrophages pour déterger les débris cellulaires intra luminaux et/ou les cristaux présents impliqués dans l'obstruction, mais aussi prévenir une agrégation cristalline plus importante du fait de leurs propriétés solubilisatrices. Enfin leur présence permettrait la formation d'un gel protéique qui pourrait favoriser la régénération tubulaire de voisinage tout en maintenant une perméabilité tubulaire et éviter ainsi la présence de "glomérules borgnes " en trop grand nombre (19). Cette prolifération tubulaire apparaît, en effet, déterminante dans les phénomènes de 
détersion et notamment dans la clairance des cristaux, permettant de recouvrir le cristal intraluminal qui se retrouve ainsi exclu de la lumière tubulaire (3). Le risque de constituer des ponts cellulaires avec les autres cellules tubulaires, sorte de synéchies qui viendraient obstruer la lumière tubulaire du néphron le rendant ainsi définitivement non fonctionnel est un événement qui semble fréquent et sous-estimé puisque survenant dans $40 \%$ des cas dans un modèle expérimental d'obstruction urétérale unilatérale (19). Le rétablissement le plus précoce possible du flux tubulaire apparaît dans cette perspective impératif afin d'empêcher ce phénomène.

\section{E) Conclusion}

L'IRA obstructive apparaît comme une entité hétérogène, la physiopathologie des obstructions intra tubulaires étant différente des obstructions aiguës des voies excrétrices. En effet dans un cas les tubules sont dilatées et le régime de pression dans les uretères, bassinets et calices est normal, dans l'autre cas, les contraintes exercées sur les parois sont importantes et entraînent une déformation des cavités rénales conduisant rapidement à une compression extrinsèque du système tubulaire avec des tubules généralement pas ou peu dilatés, les pressions intra tubulaires étant néanmoins élevées. L'IRA obstructive est cependant dans tous les cas une IRA organique, la présence de cristaux intratubulaires obstructifs et/ou de cellules desquamées et débris cellulaires et/ou gels protéiques intratubulaires entrainant une prolifération tubulaire de voisinage, un recrutement inflammatoire et dans les cas favorables, une résolution. La présence de séquelles à type de fibrose est une éventualité qui ne doit cependant pas faire sous-estimer la fréquence d'un défaut de reperméabilisation des lumières tubulaires avec constitution de nombreux néphrons non fonctionnels du fait de glomérules borgnes (i.e. atubulaires). Les phénomènes d'autorégulation de I'hémodynamique rénale ne sont donc pas nécessairement destinés uniquement à préserver un DSR et un DFG constant mais aussi à maintenir un flux tubulaire afin d'éviter l'interruption définitive du flux par une prolifération anarchique des cellules tubulaires à la suite d'un obstacle intraluminal. 
Parmi les multiples fonctions des reins, le maintien de la perméabilité tubulaire est souvent oublié, il nécessite pourtant la présence en continu de solubilisateurs tels les citrates et d'inhibiteurs macromoléculaires tels l'ostéopontine, l'acide hyaluronique ou l'uromoduline, diverses protéases, le maintien d'un pH fixe dans la plupart des segments du néphron et des variations nycthémérales importantes dans les canaux collecteurs... La présence au pôle apical de nombreuses cellules tubulaires de cils qui sont des détecteurs du flux tubulaire renforce notre conviction d'un contrôle rénal de la perméabilité tubulaire.

Conflit d'intérêt : aucun. 


\section{Références}

[1] Lionet A, Haeck M, Garstka A, Gnemmi V , Bazin D, Letavernier E, Haymann JP, Noel C, Daudon M. First investigation on microcrystalline pathologies of kidney allografts through cellular scale physicochemical techniques. CR Chimie 2016;19:1542-1548.

[2] Giebish G, Windhager E. Integration of salt and water balance. In: Boron WF, Boulpaep EL, editors. Medical physiology. Philadelphia: Saunders; 2003.

[3] Vervaet BA, Verhulst A, Dauwe SE, De Broe ME, D'Haese PC. An active renal crystal clearance mechanism in rat and man. Kidney International 2009; $75: 41-51$.

[4] Laffite G, Leroy C, Bonhomme C, Bonhomme-Coury L, Letavernier E, Daudon M, Frochot V, Haymann JP, Rouzière S, Lucas IT, Bazin D, Babonneau F, Abou-Hassan A. Calcium oxalate precipitation by diffusion using laminar microfluidics: toward a biomimetic model of pathological microcalcifications. Lab Chip. 2016;7:16(7):1157-60.

[5] Heyman SN, Rosen S, Fuchs S, Epstein FH, Brezis M. Myoglobinuric Acute Renal Failure in the Rat: A Role for Medullary Hypoperfusion, Hypoxia, and Tubular Obstruction. J. Am. Soc. Nephrol. 1996; 7:1066-1074.

[6] Vervaet BA, Verhulst A, D'Haese PC, De Broe ME. Nephrocalcinosis: new insights into mechanisms and consequences. Nephrol Dial Transplant. 2009;24(7):2030-5.

[7] El-Achkar TM, McCracken R, Liu Y, Heitmeier MR, Bourgeois S, Ryerse J, Wu XR. Tamm-Horsfall protein translocates to the basolateral domain of thick ascending limbs, interstitium, and circulation during recovery from acute kidney injury. Am J Physiol Renal Physiol. 2013 ; 304(8):F1066-75.

[8] Schaeffer C, Cattaneo A, Trudu M, Santambrogio S, Bernascone I, Giachino D, Caridi G, Campo A, Murtas C, Benoni S, Izzi C, De Marchi M, Amoroso A, Ghiggeri GM, Scolari F, Bachi A, Rampoldi L. Urinary secretion and extracellular aggregation of mutant uromodulin isoforms. Kidney Int. 2012;81(8):769-78.

[9] Sanders PW, Booker BB. Pathobiology of cast nephropathy from human Bence Jones proteins. J Clin Invest. $1992 ; 89(2): 630-9$.

[10] Arendshorst WJ, Finn WF, Gottschalk CW. Nephron stop-flow pressure response to obstruction for 24 hours in the rat kidney. J Clin Invest. 1974;53(5):1497-500.

[11] Racusen LC, Finn WF, Whelton A, Solez K. Mechanisms of lysine-induced acute renal failure in rats. Kidney Int. 1985;27(3):517-22. 
[12] Roman RJ. Altered pressure-natriuresis relationship in young spontaneously hypertensive rats. Hypertension. 1987;9(6 Pt 2):III130-6.

[13] Klahr S. Obstructive uropathy. In Brenner and Rector's The Kidney, éd, Lippincott Williams \& Wilkins. 2000. p2473-2512

[14] Pelaez LI, Juncos LA, Stulak JM, Lerman LO, Romero JC. Non-invasive evaluation of bilateral renal regional blood flow and tubular dynamics during acute unilateral ureteral obstruction. Nephrol Dial Transplant. $2005 ; 20(1): 83-8$.

[15] Vinsonneau C, Girshovich A, M'rad MB, Perez J, Mesnard L, Vandermersch S, Placier S, Letavernier E, Baud L, Haymann JP. Intrarenal urothelium proliferation: an unexpected early event following ischemic injury. Am J Physiol Renal Physiol. 2010;299(3):F479-86.

[16] Wahlberg J, Stenberg A, Wilson DR, Persson AE. Tubuloglomerular feedback and interstitial pressure in obstructive nephropathy. Kidney Int. 1984;26(3):294-301.

[17] Siegel NJ, Feldman RA, Lytton B, Hayslett JP, Kashgarian M. Renal cortical blood flow distribution in obstructive nephropathy in rats. Circ Res. 1977;40(4):379-84.

[18] Girshovich A, Vinsonneau C, Perez J, Vandermeersch S, Verpont MC, Placier S, Jouanneau C, Letavernier E, Baud L, Haymann JP. Ureteral obstruction promotes proliferation and differentiation of the renal urothelium into a bladder-like phenotype. Kidney Int. 2012;82(4):428-35.

[19] Forbes MS, Thornhill BA, Chevalier RL. Proximal tubular injury and rapid formation of atubular glomeruli in mice with unilateral ureteral obstruction: a new look at an old model. Am J Physiol Renal Physiol. 2011;301(1):F110-7. 


\section{Légendes des figures}

Figure 1. Coloration au trichrome Masson d'une coupe de rein de souris normal $(A)$ et 14 jours après une obstruction urétérale unilatérale (UUO) (B). La lumière de la veine lobaire (1) est diminuée du fait de la compression des cavités urinaires au cours de I'UUO en comparaison avec la souris contrôle alors que le calibre des artères lobaires (2) semble comparable. (C) Marquage anti BrdU (immunofluorescence) d'une coupe de rein de souris à 48 heures d'une UUO, la souris recevant du BrdU dans l'eau de boisson dés JO. Les cellules ayant proliférée au cours des 48 heures sont positives en immunofluorescence. (D) Schéma d'un rein de souris normal montrant les rapports anatomiques entre les espaces urinaires (en bleu et vert clair) et vers l'intérieur: la medullaire $(\mathrm{m})$ et la papille $(\mathrm{p})$; vers l'extérieur: le cortex $(\mathrm{c})$. La jonction cortico medullaire et medullaire externe (om) est localisée au dessus des récessus urinaires. 

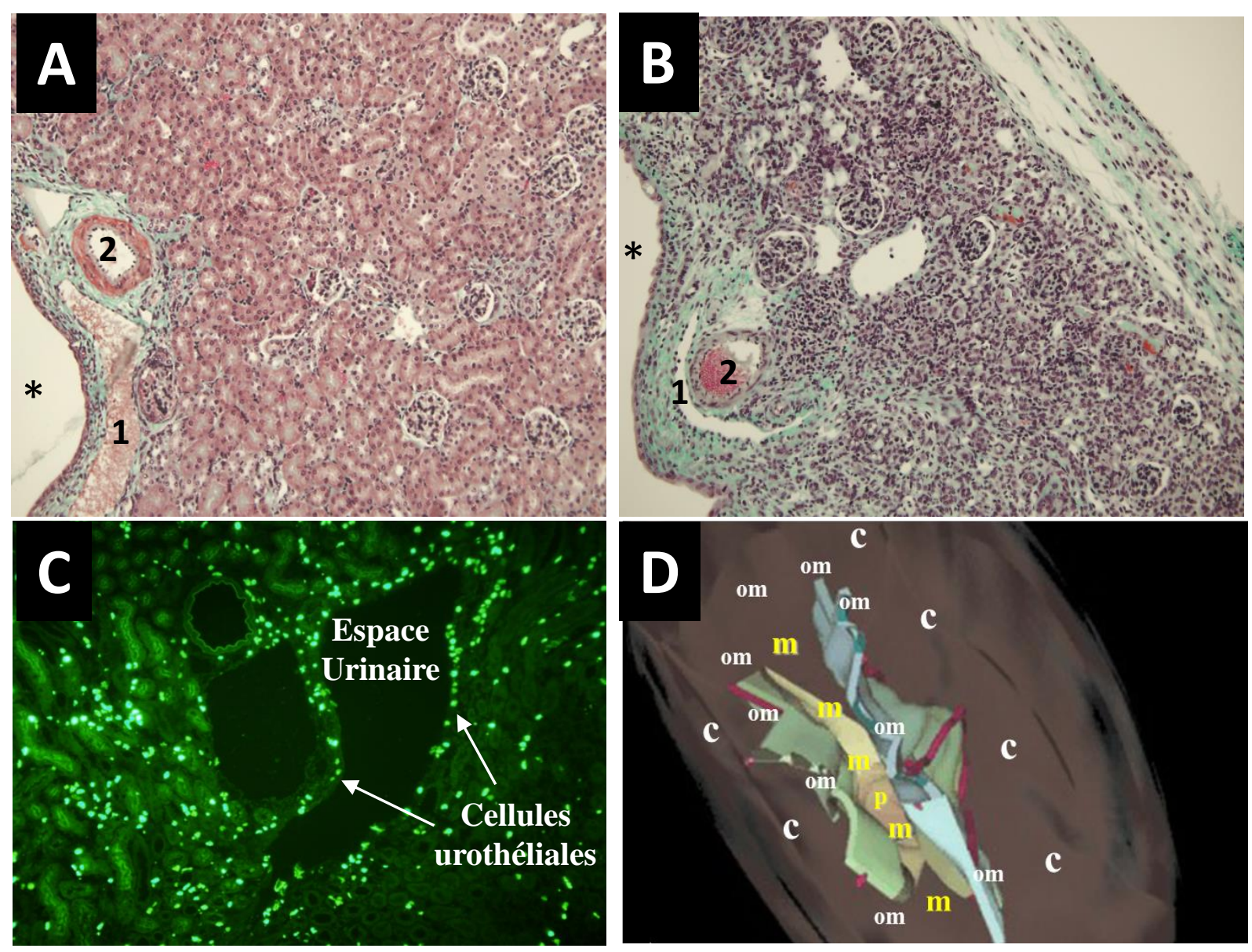

Figure 1. Coloration au trichrome Masson d'une coupe de rein de souris normal (A) et 14 jours après une obstruction urétérale unilatérale (UUO) (B). La lumière de la veine lobaire (1) est diminuée du fait de la compression des cavités urinaires au cours de l'UUO en comparaison avec la souris contrôle alors que le calibre des artères lobaires (2) semble comparable. (C) Marquage anti BrdU (immunofluorescence) d'une coupe de rein de souris à 48 heures d'une UUO, la souris recevant du BrdU dans l'eau de boisson dés J0. Les cellules ayant proliférée au cours des 48 heures sont positives en immunofluorescence. (D) Schéma d'un rein de souris normal montrant les rapports anatomiques entre les espaces urinaires (en bleu et vert clair) et vers l'intérieur: la medullaire (m) et la papille (p); vers l'extérieur: le cortex (c). La jonction cortico medullaire et medullaire externe (om) est localisée au dessus des récessus urinaires. 